\title{
CHANGES IN KI67 AS A PROGNOSTIC FACTOR AFTER NEOADJUVANT CHEMOTHERAPY IN BREAST CANCER
}

Osvaldo de Alcântara Braga Aidar ${ }^{1}$

'Universidade Federal de Goiás, Faculdade de Medicina - Goiânia (GO), Brazil.

Objective: Patients with tumors that require neoadjuvant chemotherapy may have a better prognosis when there is a good response to therapy. Those who do not have a complete response are the target of studies that aim to improve overall survival, and for that, factors that guide additional therapy should be identified. This study aims to evaluate the prognostic influence of Ki67 and its variation between the values analyzed before neoadjuvant chemotherapy and after surgery in patients with residual disease. Methods: The medical records of 126 patients treated between 2008 and 2013 at CORA/HC-UFG with breast cancer were retrospectively analyzed. Of these, 43 patients with invasive breast carcinoma met the inclusion criteria, and the data were collected on the histological and immunohistochemical types, presence of hormone receptors for invasive breast tumors, in addition to the evaluation of age, stage, and chemotherapy medications used. Ki67 should be evaluated in the material of the diagnostic core biopsy and in the surgical specimen with residual disease after neoadjuvant chemotherapy. The monitoring of events was carried out until the cutoff date of January 1, 2019. Results: The high Ki67 value at diagnosis was related to a worse prognosis, while low values were related to a lower incidence of clinical events $(p=0.004)$. The optimal value found in the receiver operating characteristic curve as a cutoff for high or low values was $25 \%$, with the statistical significance for sensitivity and specificity ( $p=0.008)$. There was no statistical significance in event-free survival and overall survival related to the Ki67 variation assessed on biopsy and surgical specimen after neoadjuvant chemotherapy, with $\mathrm{p}=0.67$ and $\mathrm{p}=0.57$, respectively. Conclusion: The high rates of Ki67 at diagnosis are related to worse survival in patients who have undergone neoadjuvant chemotherapy and have residual disease. The variation of its values before and after neoadjuvant chemotherapy cannot be used as a predictive factor to the treatment until there are larger studies, with the standardization of its evaluation.

Keywords: Breast Cancer; Ki67; Neoadjuvant Chemotherapy; Complete Pathological Response; Residual Disease. 